\title{
The features of the collective modes in aerogels filled with superfluid helium
}

\author{
A.A. Zadorozhko, V.K. Chagovets, E.Ya. Rudavskii, and G.A. Sheshin \\ B. Verkin Institute for Low Temperature Physics and Engineering of the National Academy of Sciences of Ukraine \\ 47 Lenin Ave., Kharkov 61103, Ukraine \\ E-mail: zadorozhko@ilt.kharkov.ua
}

Sh.E. Kekutia and N.D. Chkhaidze

Institute of Cybernetics, 5 Sandro Euli, Tbilisi 0186, Georgia

N. Mulders

Department of Physics and Astronomy of Delaware, Newark, Delaware 19716, USA

Received April 29, 2009

\begin{abstract}
The velocity of fast and slow collective modes of 90,94 and $98 \%$ porosity aerogels filled with superfluid helium were measured by means of low-frequency resonant technique at temperatures $0.5-2.5 \mathrm{~K}$. The temperature dependences of velocities of both modes are compared with the hydrodynamic theory which was modified taking into account the mobility of the aerogel matrix, porosity of media and tortuosity of an acoustic way. It has been found that the fast and slow modes in an aerogel are coupled much stronger than the first and second sounds in bulk He II.
\end{abstract}

PACS: 67.25.dg-Transport, hydrodynamics, and superflow; 67.25.dr-Restricted geometries; 67.25. $d t-$ Sound and excitations.

Keywords: superfluid helium, aerogel, acoustic collective modes.

\section{Introduction}

Porous substances filled with superfluid helium exhibit abundant and widely diverse wave processes. Their character is dependent on the relation between the size of pores or channels $d$ and the viscous penetration depth $\lambda_{\eta}=\left(\eta_{n} / \pi f \rho_{n}\right)^{1 / 2}\left(\eta_{n}, \rho_{n}\right.$ are the viscosity and the density of the normal He II component, respectively; $f$ is the oscillation frequency). If a porous substance is absolutely rigid (cannot participate in the oscillatory motion of the liquid) and $d<<\lambda_{\eta}$, the normal component of He II is completely clamped and the sound propagates along the superfluid component $\rho_{s}$. Such oscillations called fourth sound are actually a superposition of the first sound, the second sound and viscous waves. Pure first and second sound can propagate under another limiting condition $d>>\lambda_{\eta}$. In this case the first sound is excited by pressure oscillations and the second sound is generated by temperature oscillation. At $d \sim \lambda_{\eta}$ a sound mode occurs, which is intermediate between the first and the fourth sounds. The second sound transforms into a strongly decaying heat wave (e.g., see [1]).

In porous media the sound velocity is also dependent on the degree of channel regularity. In irregular channels the acoustic length differs from the detector-radiator spacing. In this case a correction index must be introduced into sound velocity calculation to allow for the channel tortuosity caused by multiple scattering in porous media.

The situation is very different when superfluid helium fills a porous medium of low rigidity and high compliance in which the motion of the walls and the normal components is significant. As an example let us consider an aerogel. When sound propagates in an aerogel its lattice structure is dragged by the oscillations of the normal component when $d<<\lambda_{\eta}$. The wave processes in such systems were first investigated in aerogels of high (over 95\%) porosity [2]. Two oscillatory modes were reg- 
istered in ${ }^{4} \mathrm{He}$ in aerogel from $1.2 \mathrm{~K}$ to the lambda transition temperature $T_{\lambda}$. In some experiments piezoelectric transducers were used to generate pressure waves. In this case the observed mode had a velocity intermediate between the first and the fourth sound velocities $\left(C_{1}\right.$ and $C_{4}$, respectively). A second sound type wave was excited with a heater-thermometer arrangement. The observed wave processes were defined as a fast mode and slow mode, respectively. The mode velocities were calculated using a system of equations for two-fluid hydrodynamics of He II [3] which was modified [2] to allow for the mobility of the aerogel matrix.

The general phenomenological theory of sound propagation in porous media filled with a liquid [4] was developed by Biot. Afterwards it was extended to a porous medium-superfluid helium system [5] and then to a three-component (aerogel-superfluid component-normal component) case taking into account the tortuosity of channels [6]. Using the derived equations the propagation of the longitudinal and transverse waves was analyzed within the low- and high-frequency limits in a wide range of temperatures.

The wave processes in He II-filled aerogels differ significantly from those in bulk helium. In the aerogel system both temperature and pressure oscillations are important for slow (second sound type wave) and fast (first sound type wave) modes [7]. This resembles superfluid ${ }^{3} \mathrm{He}-{ }^{4} \mathrm{He}$ solutions [1] and allows a variety of methods to be used to excite simultaneously both fast and slow modes.

The temperature dependences of the fast mode velocity and absorption were investigated in the high-frequency region on aerogels with $92.6-94.8 \%$ porosity $[8,9]$. As analysis shows, the results obtained agree with Biot's theory in normal He I and deviate appreciably in superfluid He II.

Since the pressure and temperature waves are strongly coupled in the He II-aerogel system, it is interesting to investigate the wave processes, when the slow and fast modes are excited with one transducer. Owing to the strong correlation between the porosity and the sound velocity $C_{a}$ in a «dry» aerogel, it is possible to observe how the fast and slow modes transform on changing from the case $C_{a}>C_{1}$ in dense aerogels to the case $C_{a}<C_{1}$ in a high-porosity aerogel. In this paper the densities of the aerogels permitted both cases. The fast and slow modes were excited with the same acoustic transducers. The results obtained were analyzed taking into account the tortuosity and compared with current theories $[2,6]$.

\section{Experimental technique}

The velocities of the fast and slow modes were measured by a low-frequency resonance method in the frequency range $100 \mathrm{~Hz}-20 \mathrm{kHz}$. The experimental cell (similar to the one in Ref. 10) was a copper body with an internal cylindrical hole about $8 \mathrm{~mm}$ in diameter and two caps at the ends. The diameter was selected so that the aerogel sample could fit tightly in the body. The porosity of the aerogels was 90,94 and $98 \%$. Some sample parameters are shown in Table 1. To provide good mechanical contact between the transducer and the aerogel, the body was made $0.5 \mathrm{~mm}$ shorter than the sample, which was thus slightly compressed when the cell was closed. A piezoelectric transducer with a silvered surface $(0.5 \mathrm{~mm}$ thick and $6 \mathrm{~mm}$ in diameter) was fixed to the $0.4 \mathrm{~mm}$-thick membrane at the center of each cap. The electrical connections to the transducers was made using silver paint.

Table 1. Basic characteristics of the used aerogels: porosity $\varphi$, density $\rho_{a}$, longitudinal sound velocity $C_{a}$, tortuosity $\alpha_{\infty}$ calculated from the velocities $C_{f}$ and $C_{s}$ of the fast and slow modes, respectively

\begin{tabular}{c|c|c|c|c}
\hline \hline$\varphi, \%$ & $\rho_{a}, \mathrm{~g} / \mathrm{cm}^{3}$ & $C_{a}, \mathrm{~m} / \mathrm{s}$ & $\alpha_{\infty}\left(C_{f}\right)$ & $\alpha_{\infty}\left(C_{s}\right)$ \\
\hline \hline 90 & 0.225 & 505 & $1.14 \pm 0.01$ & $1.14 \pm 0.01$ \\
94 & 0.133 & 146 & $1.07 \pm 0.01$ & $1.07 \pm 0.01$ \\
98 & 0.04 & 50 & $1.06 \pm 0.01$ & - \\
\hline \hline
\end{tabular}

The resonance curves were taken as follows. An exciting signal from a Wavetech 29 A generator was fed to one of the transducers. The transmitted signal was registered by the other transducer and fed to the input of a two-phase lock-in analyzer EG\&G 5208. The resonance curves were obtained through frequency scanning and the resonance frequencies were estimated at the signal maximum. The accuracy of the resonance frequencies was $\pm 0.5 \mathrm{~Hz}$.

The temperature range of the measurement was $0.5-2.5 \mathrm{~K}$. The acoustic cell was fixed to the ${ }^{3} \mathrm{He}$ pot of the evaporating ${ }^{3} \mathrm{He}$ refrigerator via a mechanical thermal contact. The temperature of the evaporating pot and the cell were stabilized and measured using calibrated $\mathrm{RuO}_{2}$ resistance thermometers. The accuracy of temperature measurement was $\pm 10^{-3} \mathrm{~K}$.

The cell was first cooled to low temperature and the sound velocity was measured in a «dry» aerogel (see Table 1). The helium was then condensed and the oscillation spectrum of the aerogel-He II system was registered at different temperatures. The temperature of the cell was stabilized for about $20 \mathrm{~min}$ at each point of measurement.

The typical resonance spectrum taken on one of the aerogel samples filled with superfluid helium is shown in Fig. 1. There are three peaks of the slow mode and one peak of the fast mode. The resonance peaks were identified by analyzing their temperature evolution. Resonances 1, 2 and 4 that vanished at the lambda point of the He II-He I transition corresponded to the slow mode. Peak 3 of the fast mode persisted in the He I region. 


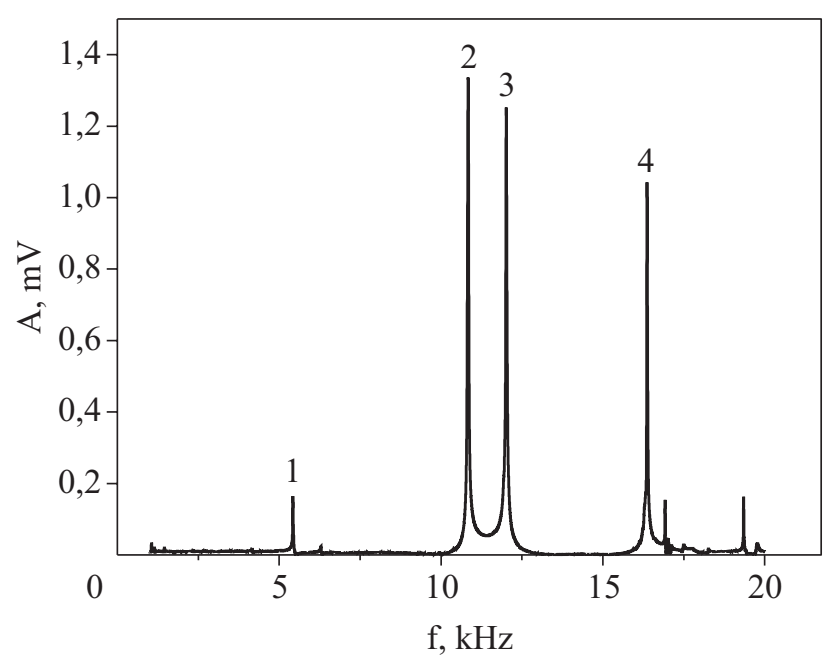

Fig. 1. The amplitude-frequency characteristic of the He II-aerogel $90 \%$ system at $T=0.7 \mathrm{~K}$.

\section{Results and discussion}

The temperature dependences of the slow and fast mode velocities measured in aerogels of 90, 94 and $98 \%$ porosity are shown in Figs. 2. The velocities $C(T)$ of both the modes were found using a simple relation

$$
C(T)=2 L f(T),
$$

where $L$ is the geometric length of the resonator, $f$ is the resonance frequency of the mode. In bulk ${ }^{4} \mathrm{He}$ the coupling between pressure and temperature oscillations in the first and the second sounds is specified by the thermal expansion coefficient, which is rather low. However, the situation changes if He II contains admixtures of ${ }^{3} \mathrm{He}$ [1] or if it is placed in aerogel [7]. In the last case, due to oscillation of either the admixture concentration or aerogel strands the coupling arise between the temperature and the pressure oscillation. The effect becomes more pronounced with increase of admixture concentration ${ }^{3} \mathrm{He}$ or aerogel density. This is clearly seen from the following relation between the pressure oscillation and temperature oscillation in the slow mode [11]:

$$
\left(\frac{P^{\prime}}{T^{\prime}}\right)_{\text {slow }}=\frac{\rho_{s} \rho_{a} \sigma}{\rho_{n}} \frac{1}{1+\frac{\rho_{a} \rho_{s}}{\rho_{n} \rho}} \frac{C_{a}^{2}}{C_{2}^{2}} .
$$

Here $\sigma$ is the entropy of the unit mass of He II. Note, that in the actual experimental condition we could not observe the slow mode in the $98 \%$ aerogel used in this experiment. Basing on above discussion, one supposes that $98 \%$ aerogel does not provide an effective coupling between pressure and temperature oscillations.

It is interesting to compare our results with the hydrodynamic calculation [2] for a two-liquid He II model allowing for the movement of the aerogel matrix during wave propagation in a high-porosity aerogel-He II system. The two-fluid hydrodynamic equations was supplemented with an equation of aerogel oscillations [2] and the extra inertia of normal fluid due to matrix. The secular equation of the sound velocity was obtained:

$$
\left(C^{2}-C_{1}^{2}\right)\left(C^{2}-C_{2}^{2}\right)+\frac{\rho_{a}}{\rho_{n}}\left(C^{2}-C_{4}^{2}\right)\left(C^{2}-C_{a}^{2}\right)=0 .
$$

The numerical solution of Eq. (3) gives the velocities of the fast $C_{f}$ and the slow $C_{s}$ modes in the aerogels of three porosities at different temperatures (see Figs. 2,
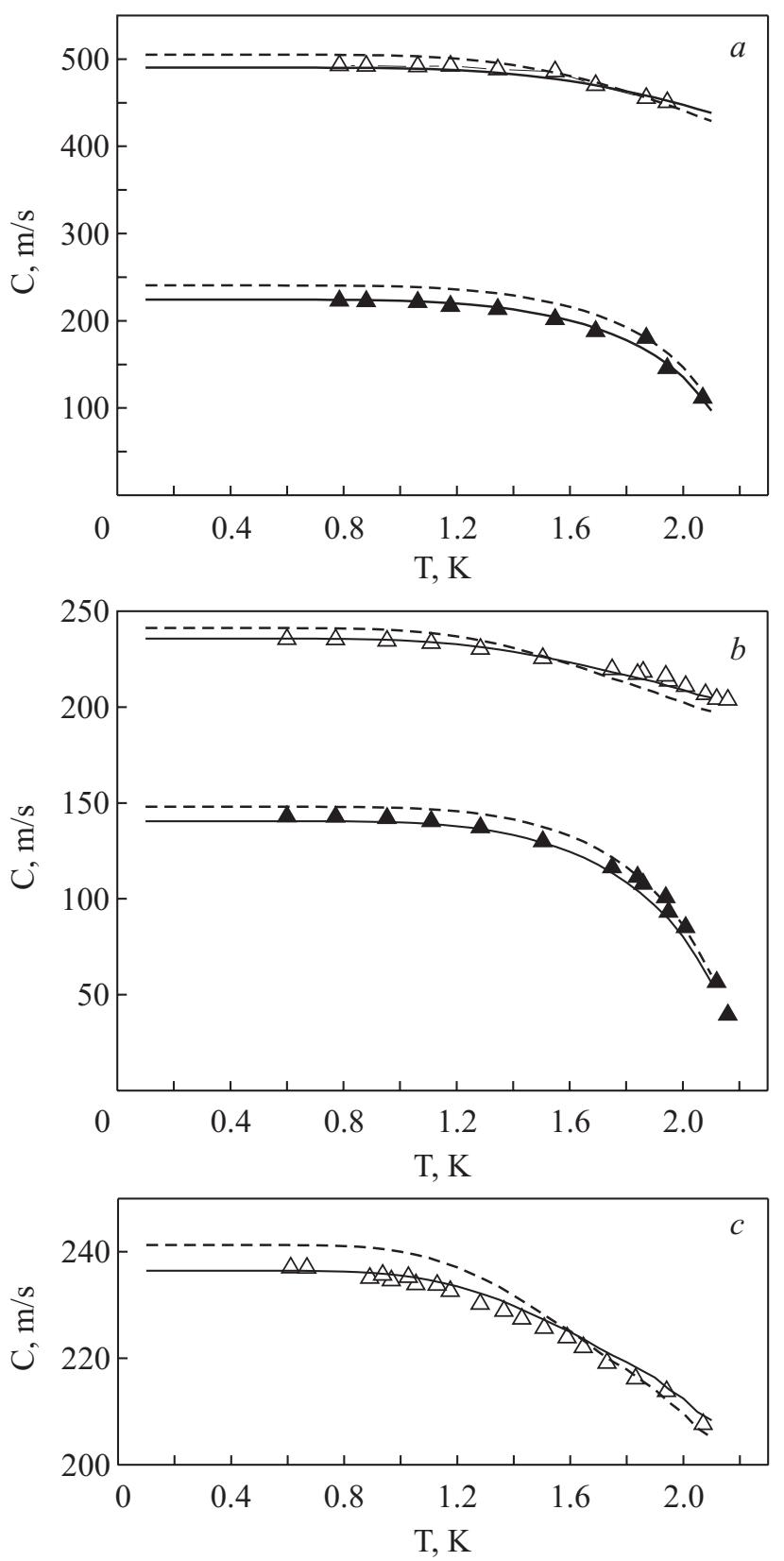

Fig. 2. The temperature dependence of the fast $(\Delta)$ and slow (A) mode velocities in the He II-aerogel (90\% (a), 94\% (b), and $98 \%(c)$ porosity) system. Calculation is made using the data of [6] (solid line) and [2] (dashed line). 
dashed lines). It is seen that the description of the dependences $C_{f}(T)$ and $C_{s}(T)$ is rather inaccurate in the whole range of temperatures and the discrepancy between experiment and calculation increases as the aerogel porosity decreases. This is due in part because the approach of Ref. 2 ignores the tortuosity as well as the porosity of the aerogel. It is inadequate to describe an aerogel whose structure is a tree network of clustered nanoparticles of $2-5 \mathrm{~nm}$ in size and pores up to $100 \mathrm{~nm}$. In Ref. 2 the agreement with experimental results was obtained only for a narrow temperature range by introducing corrections. The fast mode in the $95 \%$ porous aerogel in the range $1.3-1.8 \mathrm{~K}$ was fixed by normalization to $\rho_{s} / \rho$ at $T=1 \mathrm{~K}$. The correction for the slow mode in the sample of $99 \%$ porosity around $T_{c}$ allows for the tortuosity of the sound path.

The general analysis of the aerogel-He II system should take into account the tortuosity and the matrix porosity. These factors have been considered explicitly in a recently developed theory [6]. Its general description of the collective mode propagation in an aerogel filled with superfluid helium is based on Biot's approach [4]. The main parameters in the theory are tortuosity $\alpha_{\infty}$, porosity $\varphi$, fluid density $\rho$, aerogel density $\rho_{a}$, bulk modulus of the liquid and the aerogel, shear modulus of the aerogel. Tortuosity $\alpha_{\infty}$ is a geometrical quality, independent of the density of the solid and the density of the liquid, and can vary from 1 (for plane-parallel capillaries) to $\infty$ (for isolated pores or pores oriented perpendicular to the motion). The following secular equation was obtained in terms of this theory for fast and slow modes in the low-frequency limit $d<<\lambda_{\eta}[6]$ :

$$
\begin{gathered}
\varphi \rho_{s}\left[\alpha_{\infty} \rho_{a}+\varphi\left(\alpha_{\infty} \rho-\rho_{s}\right)\right] C^{4}- \\
-C^{2}\left\{R^{S}\left(\rho_{a}+\varphi \rho\right)+\varphi \rho_{s} \alpha_{\infty}(A+2 N+2 Q+R)-\right. \\
\left.-2 \varphi \rho_{s}\left(\frac{\rho_{s}}{\rho} Q+R^{S}+R^{S n}\right)\right\}+ \\
+R^{S}(A+2 N+2 Q+R)-\left(\frac{\rho_{s}}{\rho} Q+R^{S}+R^{S n}\right)^{2}=0,
\end{gathered}
$$

where the combinations of the parameters for the aerogel in Eq. (4) are

$$
\begin{gathered}
R^{S}=\frac{\rho_{s}^{2}}{\rho} \varphi C_{1}^{2}+\frac{\varphi \rho_{s} \rho_{n}}{\rho} C_{2}^{2}, \\
A+2 N+2 Q+R=\rho_{a} C_{a}^{2}+\frac{\rho C_{1}^{2}}{\varphi}, \\
\frac{\rho_{S}}{\rho} Q+R^{S}+R^{S n}=\rho_{S} C_{1}^{2} .
\end{gathered}
$$

The final secular equation in the low-frequency limit of oscillations is

$$
\begin{gathered}
{\left[\alpha_{\infty} \rho_{a}+\varphi\left(\alpha_{\infty} \rho-\rho_{S}\right)\right] C^{4}-} \\
-C^{2}\left[\left(\frac{\rho_{S}}{\rho} C_{1}^{2}+\frac{\rho_{n}}{\rho} C_{2}^{2}\right)\left(\rho_{a}+\varphi \rho\right)+\right. \\
\left.+\alpha_{\infty}\left(\rho_{a} C_{a}^{2}+\frac{\rho C_{1}^{2}}{\varphi}\right)-2 \rho_{S} C_{1}^{2}\right]+ \\
+\rho_{a} C_{a}^{2}\left[\frac{\rho_{S}}{\rho} C_{1}^{2}+\frac{\rho_{n}}{\rho} C_{2}^{2}\right]+\frac{\rho_{n} C_{1}^{2} C_{2}^{2}}{\varphi}=0 .
\end{gathered}
$$

Note that at $\alpha_{\infty}=\varphi=1$ Eq. (5) turns into Eq. (3).

The root of Eq. (5) the larger root of the secular equation corresponds to the fast mode velocity $C_{f}$, the smaller one describes the slow mode velocity $C_{s}$. The tortuosity index $\alpha_{\infty}$ in Eq. (5) was found by normalizing the experimental values of $C_{f}$ and $C_{S}$ to the results of calculation by Eq. (5) at $T=0.6 \mathrm{~K}$ where the velocities of both the modes are temperature-independent. Note that the values of $\alpha_{\infty}$ obtained for the fast and slow modes in an aerogel of the same porosity are very close, which complies with the conclusions in Ref. 12.

The dependences $C_{s}(T)$ and $C_{f}(T)$ were calculated by Eq. (5) for the aerogels with 90,94 and $98 \%$ porosity using the $\alpha_{\infty}$ values (see Figs. 2, solid lines). It is seen that the theory [6] describes the measured velocities of the two modes quite accurately in the whole temperature range.

\section{The effect of aerogel porosity on collective mode velocities}

As follows from experimental data, the velocities of both fast and slow modes are strongly dependent on the porosity of aerogels. This is clearly seen in Fig. 3 showing $C_{s}(\varphi)$ and $C_{f}(\varphi)$ calculated in terms of the theory [6] (solid lines) for two temperatures. The velocities of the first $\left(C_{1}\right)$, and fourth $\left(C_{4}\right)$ sound in He II (curves 3 and 4 , respectively) and also the sound speed in «dry» aerogel $C_{a}$ are included for comparison. At rather low temperatures (Fig. 3,a) at which the velocities $C_{s}$ and $C_{f}$ are practically temperature-independent, in the high-porosity limit $(\varphi \rightarrow 1) C_{s}$ tends to $C_{a}$ and $C_{f}$ approaches $C_{1}$ for the bulk He II.

As the aerogel density increases (porosity decreases), $C_{f}$ starts to exceed $C_{1}$ and reaches the value of the sound velocity $C_{a}$ in a «dry» aerogel. In dense aerogels $C_{S}$ tends to the fourth sound velocity $C_{4}$ which practically coincides with $C_{1}$ at low temperatures. The experimental points fall accurately onto the curves calculated by the theory developed in Ref. 6. 

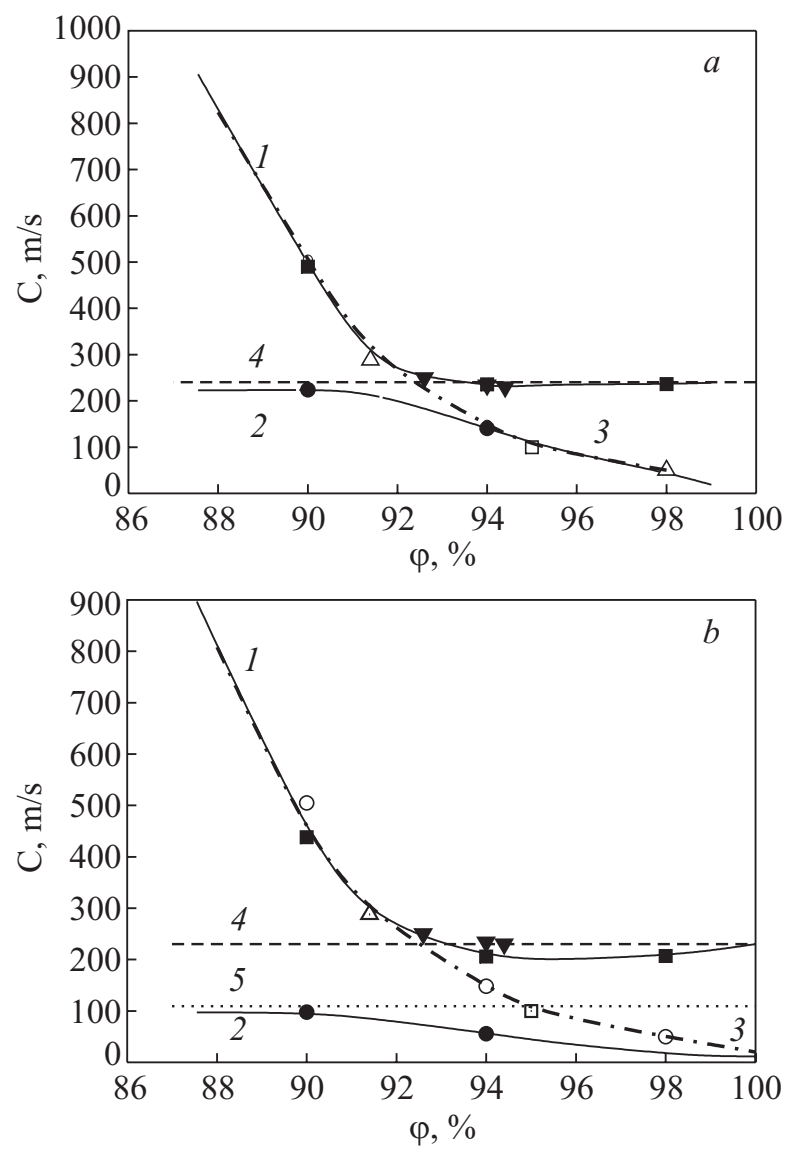

Fig. 3. The dependence of the fast (1) and slow (2) mode velocities upon the aerogel porosity: $T=0.6 \mathrm{~K}(a)$ and $2.1 \mathrm{~K}(b)$. Fast mode: this study $(\boldsymbol{\square}),[8,9](\boldsymbol{\nabla})$. Slow mode: this study (๑). Solid lines 1,2 - calculation by theory [6]. Line 3 sound velocity in «dry» aerogel, line 4 - first sound velocity, line 5 - fourth sound velocity. Sound velocity in «dry» aerogel: [13] $(\triangle)$, [2] $(\square)$, this study $(O)$.

The behavior of fast and slow modes is more intricate near the lambda temperature $T_{\lambda}$ (see Fig. $3, b$ ). In this temperature range in high-porosity aerogels $(\varphi \rightarrow 1)$ the fast mode velocity changes from value $C_{f} \approx C_{1}$ to magnitudes intermediate between $C_{1}$ and $C_{4}$, as it was in Ref. 2. As the porosity decreases further, when $C_{a}>C_{1}, C_{f}$ tends rapidly to $C_{a}$, like in the low-temperature case. At $\varphi \rightarrow 1$ the velocity $C_{s}$ approaches $C_{2}$ and in dense aerogels $C_{S}$ tends to $C_{4}$, however $C_{4}<C_{1}$ at these temperatures.

So the analysis of the limiting cases shows that for slow mode there exist two limits at $\varphi \rightarrow 1$ depending on temperature: $C_{s}(\varphi) \rightarrow \mathrm{C}_{2}$ near $T_{c}$ as is shown in Ref. 2, whereas $C_{s}(\varphi) \rightarrow \mathrm{C}_{a}$ at $T \rightarrow 0$. The reason of the dip of the fast mode is increase of the normal component of superfluid helium. Especially, this phenomena is caused by the parameter $1+\rho_{a} / \rho_{n}$. Since the sound velocity increases sharply with aerogel density, we can assume, that in dense aerogels fast modes travel mainly through aerogel matrices («dense» means $\varphi<93 \%$ ).

Note, that in Ref. 14 where $90 \%$ aerogel was studied the experimental condition did not allow to measure the sound velocity in the «dry» aerogel. For this reason the underestimated value of $C_{a}$ was applied in data analysis. As a result the slow mode was erroneously taken for the fast one.

\section{Conclusions}

In this series of experiments the velocities of the fast and slow collective modes in aerogels of different porosities filled with He II have been investigated in a wide range of temperatures. The measurements were made in the low-frequency region where the viscous penetration depth exceeds the characteristic size of the pores in the aerogel. Under this condition, the normal component of He II is completely clamped in a rigid porous medium and can not take part in propagation of oscillations. In an aerogel the normal component moves dragging the aerogel strands. The activity of the aerogel in propagation of the wave is dependent on its density. The results obtained illustrate how the character of the wave processes changes with the aerogel porosity.

The analysis provided shows that for an adequate description of experimental data, one should take into account the irregularity of the channels which is equivalent to introduce the tortuosity coefficient. Such an approach, developed in Ref. 6, allowed to achieve a rather good agreement with experiment. Note, hydrodynamic theory $[2,6]$ can be used to analyse the experimental data if wave length is larger than both the channel size and mean free path of the quasiparticles.

It has been found that the fast and slow modes in an aerogel are coupled much stronger than the first and second sounds in bulk He II. This is supported by the excitement of a slow mode by pressure oscillations.

The work is partially supported with a STCU grant (Project 3718).

1. M.I. Kaganov, B.N. Eselson, E.Ya. Rudavskii, and I.A. Serbin, Usp. Fiz. Nauk 112, 591 (1974).

2. M.J. McKenna, T.M. Slawecki, and J.D. Maynard, Phys. Rev. Lett. 66, 1878 (1991).

3. M.I. Khalatnikov, Theory of Superfluidity, Nauka Publ., Moskov (1971).

4. A.M. Biot, J. Acoust. Soc. Am. 28, 168 (1956); ibid. 28, 179 (1956).

5. D.L. Johnson, Appl. Phys. Lett. 37, 1065 (1980).

6. Sh.E. Kekutia and N.D. Chkhaidze, Fiz. Nizk. Temp. 28, 1115 (2002) [Low Temp. Phys. 28, 795 (2002)]; Sh.E. Kekutia and N.D. Chkhaidze, Journal of Statistical Mechanics: Theory and Experiment, 2005/P12008.

7. Peter Brusov, J.M. Parpia, Paul Brusov, and G. Lawes, Phys. Rev. B63, 140507 (2001). 
8. K. Matsumoto, T. Higaki, Y. Matsuyama, and K. Tajiri, Physica B329-333, 335 (2003).

9. K. Matsumoto, Y. Matsuyama, D.A. Tayurskii, and K. Tajiri, J. Exp. Theor. Phys. Lett. 80, 118 (2004).

10. D.R. Daughton, J. MacDonald, and N. Mulders, J. NonCryst. Solids 319, 297 (2003).

11. Sh.E. Kekutia and N.D. Chkhaidze, Fiz. Nizk. Temp. 32, 816 (2006) [ Low Temp. Phys. 32, 621 (2006)].
12. D. Singer, F. Pasierb, R. Ruel, and H. Kojima, Phys. Rev. B30, 2909 (1984).

13. N. Mulders, R. Mehrotra, L.S. Goldner, and G. Ahlers, Phys. Rev. Lett. 67, 695 (1991).

14. A.A. Zadorozhko, V.K. Chagovets, E.Ya. Rudavskii, T.V. Kalko, G.A. Sheshin, and N. Mulders, J. Low Temp. Phys. 150, 505 (2008). 